\title{
Promotion of Remyelination by Sulfasalazine in a Transgenic Zebrafish Model of Demyelination
}

\author{
Suhyun Kim ${ }^{1,4}$, Yun-II Lee, ${ }^{2,4}$ Ki-Young Chang ${ }^{2}$, Dong-Won Lee ${ }^{1}$, Sung Chun Cho ${ }^{2}$, Young Wan $\mathrm{Ha}^{2}$, \\ Ji Eun $\mathrm{Na}^{3}$, Im Joo Rhyu', Sang Chul Park ${ }^{2 *}$, and Hae-Chul Park ${ }^{1, *}$
}

\begin{abstract}
Most of the axons in the vertebrate nervous system are surrounded by a lipid-rich membrane called myelin, which promotes rapid conduction of nerve impulses and protects the axon from being damaged. Multiple sclerosis (MS) is a chronic demyelinating disease of the CNS characterized by infiltration of immune cells and progressive damage to myelin and axons. One potential way to treat MS is to enhance the endogenous remyelination process, but at present there are no available treatments to promote remyelination in patients with demyelinating diseases.

Sulfasalazine is an anti-inflammatory and immunemodulating drug that is used in rheumatology and inflammatory bowel disease. Its anti-inflammatory and immunomodulatory properties prompted us to test the ability of sulfasalazine to promote remyelination. In this study, we found that sulfasalazine promotes remyelination in the CNS of a transgenic zebrafish model of NTR/MTZ-induced demyelination. We also found that sulfasalazine treatment reduced the number of macrophages/microglia in the CNS of demyelinated zebrafish larvae, suggesting that the acceleration of remyelination is mediated by the immunomodulatory function of sulfasalazine. Our data suggest that temporal modulation of the immune response by sulfasalazine can be used to overcome MS by enhancing myelin repair and remyelination in the CNS.
\end{abstract}

\section{INTRODUCTION}

Most axons in the vertebrate nervous system are surrounded by a lipid-rich membrane called myelin, which promotes rapid

\footnotetext{
${ }^{1}$ Department of Biomedical Sciences, Korea University, Ansan 425-707, Korea, ${ }^{2}$ Well Aging Research Center, Samsung Advanced Institute of Technology (SAIT), Suwon 443-803, Korea, ${ }^{3}$ Department of Anatomy, College of Medicine, Korea University, Seoul 136-705, Korea, ${ }^{4}$ These authors contributed equally to this work.

*Correspondence: hcpark67@korea.ac.kr (HCP); sc2013.park@samsung. com (SCP)
}

Received 15 September, 2015; revised 11 October, 2015; accepted 13 October, 2015; published online 6 November, 2015

Keywords: dorsal midline, enhancer, EphA7 conduction of nerve impulses and protects against axonal damage. During development, myelin is produced by oligodendrocytes in the CNS and Schwann cells in the PNS (Emery, 2010). In addition to its role in forming myelin, a recent study has shown that oligodendrocytes provide metabolic support to neurons, which is crucial for axonal function and neuronal survival (Lee et al., 2012). Demyelination is the pathological process by which the myelin sheath is lost from around the axon. Normally, the CNS has the capacity to repair demyelinated axons through a process called remyelination, in which new myelin is synthesized to remyelinate demyelinated axons, enabling them to recover lost function. However, myelin is essential, and disruption of the myelin structure and failure to remyelinate in the CNS causes various neurodegenerative diseases including multiple sclerosis (MS).

MS is a chronic demyelinating disease of the CNS characterized by infiltration of immune cells and progressive damage to myelin and axons. MS is thought to be caused by the immune system inappropriately attacking the myelin in the brain and spinal cord, resulting in the demyelination of axons (Franklin, 2002; Franklin and Ffrench-Constant, 2008). It has been proposed that the enhancement of the endogenous remyelination process is one of the potential ways to efficiently treat MS, and several animal models of chemically induced demyelination such as cuprizone and lysolecitin have been used to screen drug candidates to promote remyelination (Imai et al., 2008; Lau et al., 2012; Moharregh-Khiabani et al., 2010; Ransohoff, 2012; Silvestroff et al., 2012). Although considerable progress has been achieved to promote remyelination in the demyelinated CNS, there are no available treatments to promote remyelination in patients with demyelinating diseases.

To investigate remyelination in vivo, we previously generated an animal model of demyelination using a transgenic zebrafish system in which a bacterial cytotoxin gene, $n f s B$, which encodes a nitroreductase (NTR) enzyme that can convert prodrugs such as metronidazole (MTZ) into cytotoxins, is expressed in oligodendrocytes under the control of the mbp promoter (Chung et al., 2013). Exposure of transgenic zebrafish to an MTZ-containing medium resulted in rapid ablation of oligodendrocytes and subsequent demyelination of the CNS in both larvae and adults (Chung et al., 2013). In this study, we tested whether sulfasalazine treatment promotes remyelination in the CNS of a transgenic zebrafish model of NTR/MTZ-induced demyelination. Sulfasalazine is an anti-inflammatory 


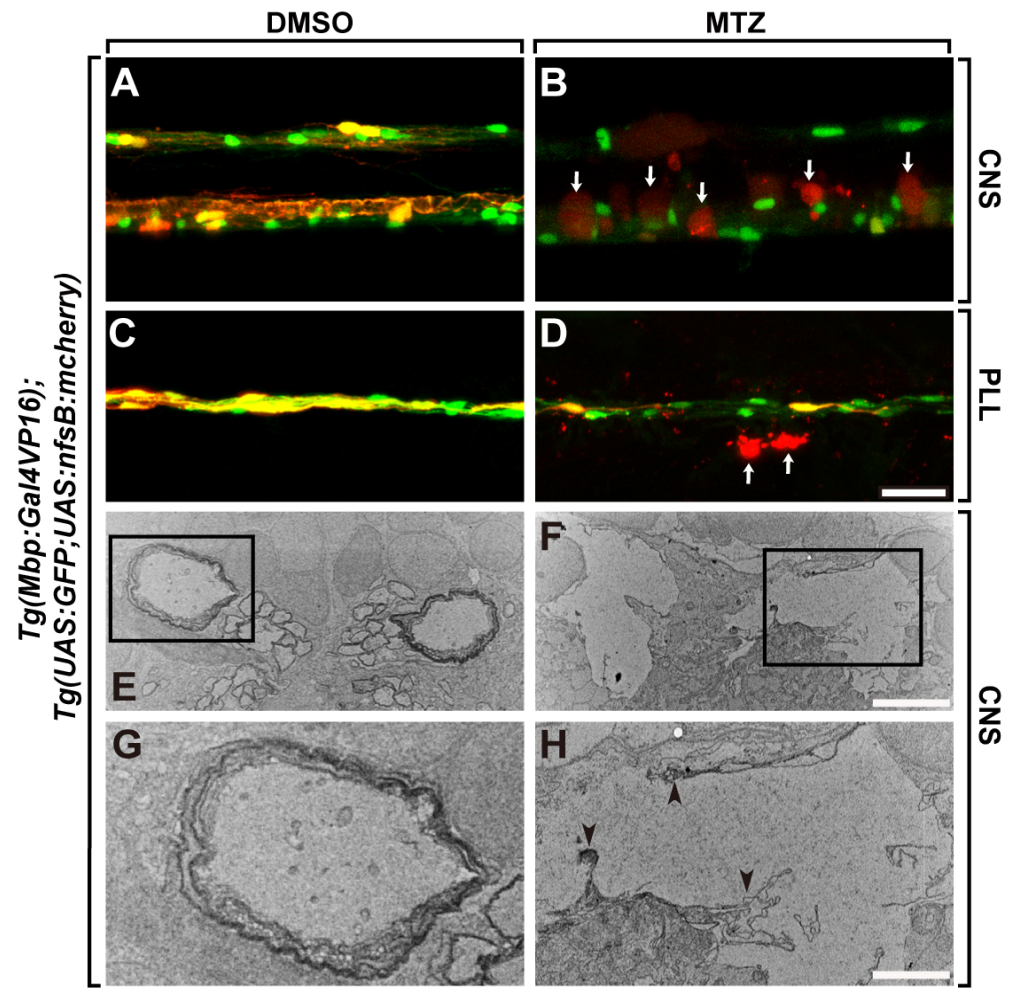

Fig. 1. Demyelination in the zebrafish nervous system by targeted ablation of myelinating glial cells. (A-D) Lateral view of the spinal cord of Tg(mbp:gal4-vp16;uas:nfsB-mcherry;uas:gfp) larvae with anterior to the left and dorsal to the top. Transgenic larvae were treated with $\operatorname{DMSO}(\mathrm{A}, \mathrm{C})$ and MTZ (B, D) at $5 \mathrm{dfp}$ for three days. Arrows indicate myelin debris in (B, D). (E-H) Transmission electron microscope images of transverse sections of the spinal cord of $T g$ (mbp:gal4-vp16;uas:nfsB-mcherry; uas:gfp) larvae treated with MTZ at $5 \mathrm{dpf}$ for three days. Normal myelin sheaths are observed surrounding Mauthner axons in the DMSO-treated control larvae $(E, G)$. The myelin sheath surrounding Mauthner axons was disrupted in MTZ-treated transgenic larvae $(F, H)$. Arrowheads indicate myelin debris in $(H) .(G, H)$ Higher magnification of the boxed area in $(E)$ and $(F)$, respectively. Scale bars: $100 \mu \mathrm{m}$ in (A-D), $5 \mu \mathrm{m}$ in (E, F), and $2 \mu \mathrm{m}$ in $(G, H)$. and immune-modulating drug that is mainly used in rheumatology and inflammatory bowel disease (Dale et al., 2007; Plosker and Croom, 2005; Zenlea and Peppercorn, 2014). Its mode of action remains elusive; however, previous reports have shown that sulfasalazine induces immunosuppressive prostaglandins, reduces the synthesis of reactive oxygen compounds, and stabilizes lipid membranes of the cell (Hoult, 1986; Peppercorn, 1984). Its anti-inflammatory and immunomodulatory properties led us to test whether sulfasalazine promotes remyelination, and we found that sulfasalazine promotes oligodendrocyte regeneration and subsequent remyelination in the CNS of a transgenic zebrafish model of NTR/MTZ-induced demyelination. Sulfasalazine treatment did not affect the proliferation of neural precursors in the CNS of wild-type embryos but promoted its proliferation in the CNS of demyelinated zebrafish larvae. Finally, we found that sulfasalazine treatment reduced the number of CNS macrophages/microglia in the CNS of demyelinated zebrafish larvae, suggesting that promotion of remyelination is mediated by the immunomodulatory functions of sulfasalazine.

\section{MATERIALS AND METHODS}

\section{Animals}

The zebrafish embryos used in this study were Tg(olig2: egfp) ${ }^{\text {vi12 }}$ (Shin et al., 2003), Tg(mbp:gal4-vp16;uas:nfsB-mcherry) (Chung et al., 2013), Tg(claudink:gal4-vp16;uas:nfsB-mcherry) (Munzel et al., 2012), and Tg(uas:egfp) (Asakawa and Kawakami, 2008). Embryos were staged according to "days post-fertilization" (dpf), hours post-fertilization (hpf)" and morphological criteria (Kimmel et al., 1995).

\section{Metronidazole and sulfasalazine treatment}

Metronidazole (Mtz, M1547, Sigma) was dissolved in EM containing $0.2 \%$ DMSO to $10 \mathrm{mM}$ as previously described (Chung et al., 2013). For the ablation of myelinating glia, $\operatorname{Tg}(\mathrm{mbp}$ :gal4-
vp16;uas:nfsB-mcherry) and Tg(claudink:gal4-vp16;uas:nfsBmcherry) larvae were incubated in $10 \mathrm{mM}$ Mtz for $72 \mathrm{~h}$ starting at $5 \mathrm{dpf}$. For sulfasalazine (Sigma) treatment, transgenic larvae were rinsed at $7 \mathrm{dpf}$ in embryo medium (EM; $15 \mathrm{mM} \mathrm{NaCl}, 0.5$ $\mathrm{mM} \mathrm{KCl}, 1 \mathrm{mM} \mathrm{CaCl} 2,1 \mathrm{mM} \mathrm{MgSO}_{4}, 0.15 \mathrm{mM} \mathrm{KH}_{2} \mathrm{PO}_{4}, 0.05$ $\mathrm{mM} \mathrm{NH}_{2} \mathrm{PO}_{4}, 0.7 \mathrm{mM} \mathrm{NaHCO}_{3}$ ) and incubated in EM containing 10 uM sulfasalazine for 1 day.

\section{BrdU labeling and immunohistochemistry}

Manually-dechorionated embryos were labeled with BrdU (Roche) by incubating them for $1 \mathrm{~h} 30 \mathrm{~min}$ on ice in a solution of $10 \mathrm{mM}$ BrdU and 15\% DMSO in EM at $8 \mathrm{dpf}$. The embryos were then placed in $\mathrm{EM}$, incubated for $1 \mathrm{~h}$ at $28.5^{\circ} \mathrm{C}$, and fixed using $4 \%$ paraformaldehyde in PBS. Embryos were processed for immunohistochemistry, treated for 20 min with $2 \mathrm{M} \mathrm{HCl}$, and were then processed for anti-BrdU immunohistochemistry. For immunohistochemistry, we used the following primary antibodies: mouse anti-BrdU (G3G4, 1:250, Developmental Studies Hybridoma Bank), rabbit anti-Sox10 (1:800, Lifespan bioscience), rat cd11b (1:100, BD bioscience), mouse anti-sodium channel (1:500, Sigma), and mouse anti-acetylated tubulin (1:1000, Sigma). Alexa 488-, 568-, 647-conjugated secondary antibodies were used for fluorescence detection (1:500, Molecular Probe). For histology section, embryos were embedded in $1.5 \%$ agar $/ 5 \%$ sucrose. Sections $(10 \mu \mathrm{m})$ were obtained by using a cryostat microtome (Zeiss).

\section{Fluorescent in situ hybridization}

Fluorescent in situ RNA hybridization was performed as previously described (Brend and Holley, 2009) using the Tyramide Signal Amplification method (PerkinElmer). apoe RNA probe was used to label microglia (Tedde et al., 2008).

\section{TEM imaging}

Tissues were prepared using standard procedures for TEM as 

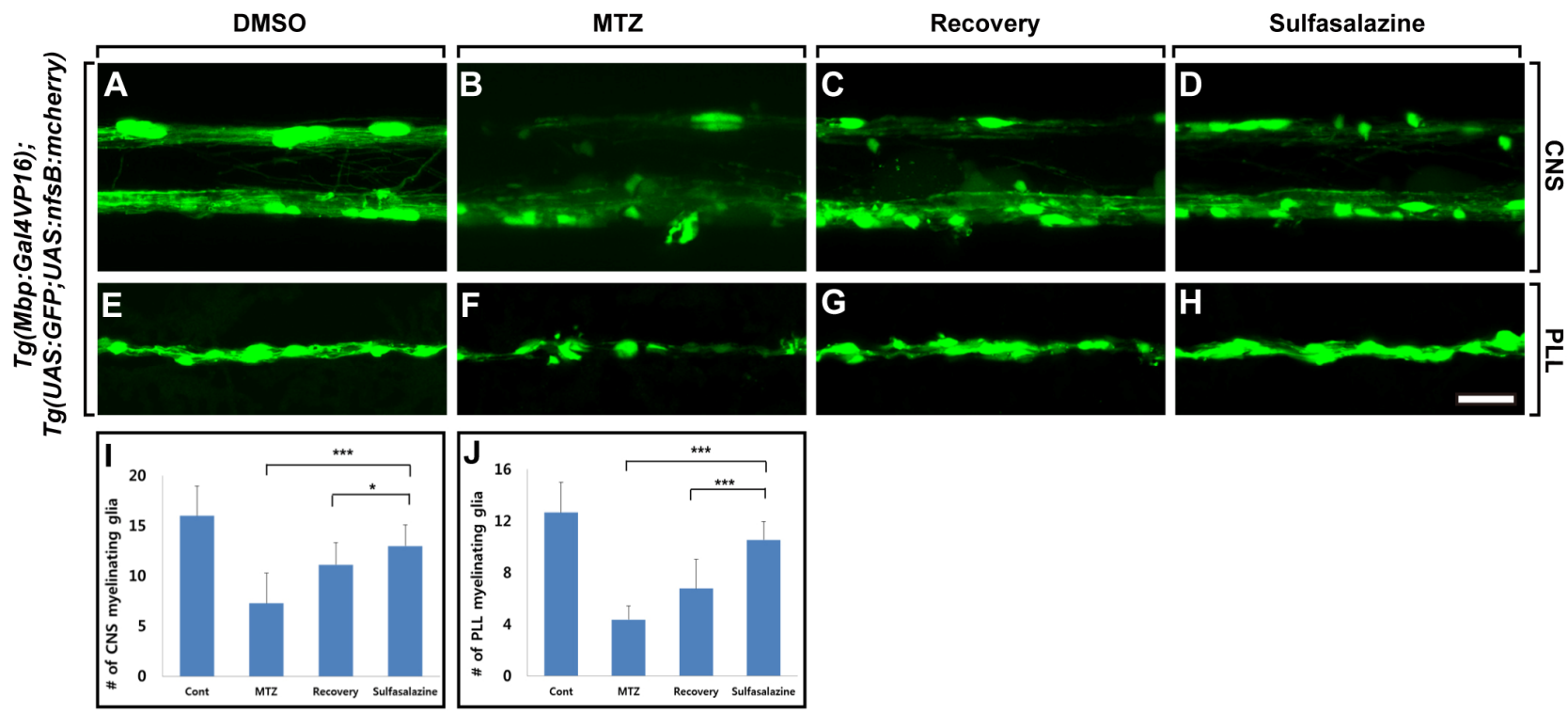

Fig. 2. Sulfasalazine promotes regeneration of oligodendrocytes and Schwann cells in the transgenic model of demyelination. All images are lateral views of the spinal cord of Tg(mbp:gal4-vp16;uas:nfsB-mcherry;uas:gfp) larvae with anterior to the left and dorsal to the top. GFP fluorescence indicates Mbp:GFP ${ }^{+}$oligodendrocytes and its myelin sheath in the spinal cord (A-D) and Schwann cells and its myelin sheath in the PLL (E-H). Transgenic larvae were treated with DMSO (A, E) and MTZ (B, F) at 5 dpf for three days and recovered for one day in EM (C, G) and sulfasalazine solution (D, H). (I, J) Quantification of the number of myelinating glia in the spinal cord of $T g(m b p: g a l 4-v p 16$; uas:nfsBmcherry;uas:gfp). Data were obtained from each of 12 transgenic larvae. Error bars represent s.d. $\left({ }^{* \star *} p<0.001,{ }^{*} p<0.05\right)$. Scale bar, $100 \mu \mathrm{m}$.

described previously (Kim et al., 2002). Briefly, zebrafish larva were anesthetized with Tricaine (Sigma) and fixed with $10 \%$ paraformaldehyde $/ 2.5 \%$ glutaraldehyde $/ 0.1 \mathrm{M}$ phosphate buffer, $\mathrm{pH}$ 7.4. Then larva were postfixed in $1 \%$ osmium tetroxide, dehydrated, embedded in Eponate-12 resin (Ted Pella). 1- $\mu$ mthick sections were obtained using Reichert-Jung Ultracut $E$ ultramicrotome (Leica), stained with toluidine blue, and imaged using a Carl Zeiss Axio microscope. 60-nm-thick sections per block were collected on Formvar-coated slot grids and stained with uranyl acetate/lead citrate, and then recorded on an $\mathrm{H}$ 7500 TEM (Hitachi) $(80$ kV).

\section{Stab wound}

Adult brain injury was performed as described previously (Marz et al., 2011). Adult zebrafish were anesthetized with Tricaine (Sigma) and a BD U-100 insulin syringe (BD bioscience) was inserted vertically through the skull into the telencephalic region. Then brain damaged fish were transferred into a tank with fresh fish water and fixed in 4\% paraformaldehyde at 4-day post injury.

\section{RESULTS}

Demyelination by targeted ablation of oligodendrocytes and Schwann cells in the zebrafish nervous system

To investigate remyelination in vivo, we previously generated an animal model of demyelination using a transgenic zebrafish system in which a bacterial cytotoxin gene, $n f s B$, which encodes a nitroreductase (NTR) enzyme that can convert prodrugs such as metronidazole (MTZ) into cytotoxins, is expressed in oligodendrocytes under the control of the mbp promoter (Chung et al., 2013).

To examine the effectiveness of the NTR/MTZ system at inducing demyelination in the CNS and PNS via drug-dependent ablation of oligodendrocyte lineage cells, we first crossed Tg(mbp:al4-vp16) driver lines with Tg(uas:egfp;uas:nfsBmcherry) fish to generate $T g$ (mbp:gal4-vp16;uas:gfp;uas:nfsBmcherry) larvae and exposed bitransgenic larvae to the prodrug, MTZ, at $5 \mathrm{dpf}$. In the absence of MTZ, bitransgenic larvae do not induce ablation of myelinating glial cells; thus, oligodendrocytes in the spinal cord (Fig. 1A) and Schwann cells in the posterior lateral line (PLL) (Fig. 1C), which express both GFP and $n f s B$-mCherry, are clearly visible in the $T g$ (mbp:gal4-vp16; as:gfp;uas:nfsB-mcherry) larvae. However, exposure of $5 \mathrm{dpf}$ Tg(mbp:gal4-vp16;uas:gfp;uas:nfsB-mcherry) larvae to $10 \mathrm{mM}$ MTZ for three days caused ablation of most of the nfsB$\mathrm{mCherry}^{+}$oligodendrocytes. Thus transgenic larvae showed several dying cells with fuzzy red fluorescence in the spinal cord (Fig. 1B, arrows) and red fluorescent cellular debris in the PLL (Fig. 1D, arrows). To confirm demyelination in the spinal cord of transgenic zebrafish, we performed transmission electron microscopy (TEM) imaging. After MTZ treatment for three days, myelin debris was present surrounding Mauthner axons of Tg(mbp:gal4-vp16;uas:gfp;uas:nfsB-mcherry), and the Mauthner axon structure was disrupted following myelin breakdown (Figs. $1 \mathrm{~F}$ and $1 \mathrm{H}$, arrows). However, wild-type larvae showed compact myelin structure around Mauthner axons and neighboring axons in the ventral spinal cord (Figs. 1E and 1G). Altogether, these data indicate that NTR/MTZ transgenic system efficiently induced demyelination in zebrafish nervous system.

Sulfasalazine promotes regeneration of myelinating glial cells and remyelination in the demyelinated zebrafish nervous system

To investigate whether sulfasalazine promotes remyelination in the demyelinated zebrafish nervous system, Tg(mbp:gal4vp16;uas:gfp;uas:nfsB-mcherry) larvae were first exposed to MTZ at 5 dfp for two days to induce demyelination by ablation 

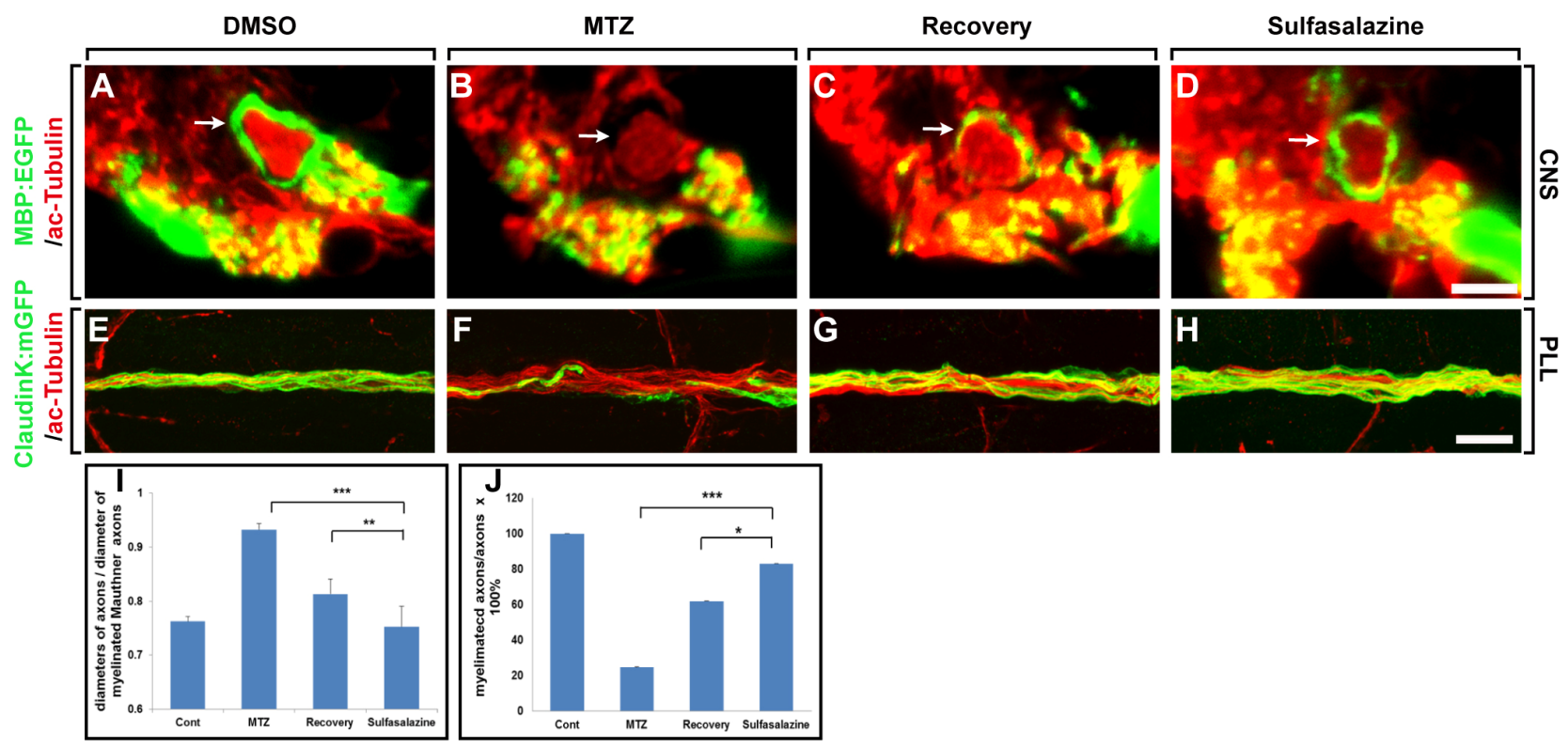

Fig. 3. Sulfasalazine promotes remyelination of demyelinated axons in the zebrafish nervous system. (A-D) Transverse sections of the spinal cord of Tg(mbp:gal4-vp16;uas:nfsB-mcherry;uas:gfp) larvae; dorsal is at the top. (E-H) Whole-mount lateral views of PLL of Tg(claudinK:gal4vp16;uas:nfsB-mcherry;uas:gfp) larvae with dorsal to the top and anterior to the left. Transgenic larvae were labeled with an anti-acetylated tubulin antibody to detect axons (red color) and green fluorescence marks the myelin sheath. Transgenic larvae were treated with DMSO (A, $E)$ and MTZ $(B, F)$ at $5 \mathrm{dpf}$ for two days and recovered for one day in EM $(C, G)$ and sulfasalazine solution $(D, H)$. Arrows indicate the myelin sheath of Mauthner axons. (I) Ratio of diameters of Mauthner axons and diameter of myelinated Mauthner axons. (J) Percentages of myelinated axons per axons in the PLL of Tg(claudink:gal4-vp16;uas:nfsB-mcherry;uas:mgfp) larvae. Data were obtained from each of six transgenic larvae. Error bars represent s.d. $\left.{ }^{* \star *} p<0.001,{ }^{* *} p<0.01,{ }^{*} p<0.05\right)$. Scale bars, $50 \mu \mathrm{m}$ in (A-D), $10 \mu \mathrm{m}$ in $(E-H)$.

of oligodendrocytes and Schwann cells. After ablation by MTZ treatment, larvae were washed out to remove MTZ and raised in EM and EM-sulfasalazine media separately to compare remyelination efficiency. After MTZ treatment, the number of GFP-expressing oligodendrocytes and Schwann cells was significantly reduced in $T g$ (mbp:gal4-vp16;uas:gfp;uas:nfsBmcherry) larvae (Figs. 2B, 2F, 2l, and 2J) compared with DMSO-treated wild-type larvae (Figs. $2 \mathrm{~A}$ and $2 \mathrm{E}$ ), but the number of oligodendrocytes and Schwann cells recovered slightly in the MTZ-treated larvae after one day of recovery time (Figs. 2C, 2G, 2I, and 2J). Interestingly, following sulfasalazine treatment, the number of oligodendrocytes and Schwann cells recovered to levels similar to those of control larvae after one day of recovery, indicating that sulfasalazine promotes regeneration of oligodendrocytes and Schwann cells (Figs. 2D, 2H, 2l, and $2 \mathrm{~J}$ ).

Next, we investigated whether sulfasalazine treatment promotes remyelination following the regeneration of myelinating glial cells in the CNS and PNS. Tg(mbp:gal4vp16;uas:gfp; as:nfsB-mcherry) larvae were used to test CNS remyelination and Tg(claudinK:gal4-vp16;uas:megfp;uas:nfsBmcherry) larvae were used to test PNS remyelination. In the ventral spinal cord of DMSO-treated control transgenic larvae, anti-acetylated tubulin antibody labeling shows Mauthner axons surrounded by intact an myelin sheath, marked by mbp:GFP fluorescence (Fig. 3A, arrow), and posterior lateral line (PLL) axon fibers surrounded by intact ClaudinK:mGFP fluorescence in the PNS (Fig. 3E, arrow). To compare remyelination efficiency quantitatively, we measured g-ratio, defined as the ratio of the inner axonal diameter to the outer myeli nated axonal diameter. Therefore high g-ratio value $(\leq 1)$ means demyelinated state of axons. After MTZ treatment, $\mathrm{GFP}^{+}$myelin sheath surrounding Mauthner axons and PLL axon fibers were disrupted following oligodendrocyte and Schwann cell ablation (Figs. 3B and 3F, arrows). However, sulfasalazine treatment promoted remyelination of Mauthner axons (Figs. 3D and $3 \mathrm{I}$ ) and PLL axon fibers (Figs. 3H and $3 \mathrm{~J})$ compared with the transgenic larvae without sulfasalazine treatment (Figs. $3 \mathrm{C}$ and $3 \mathrm{G}$ ), indicating that sulfasalazine promoted remyelination following oligodendrocyte and Schwann cell regeneration in the CNS and PNS.

To investigate whether the regenerated myelinating glial cells in the sulfasalazine-treated larvae reconstructed a functional myelin sheath in the nervous system, we asked if the nodes of Ranvier structure reformed in the regenerated myelin sheath. To do so, we used an antibody against sodium channels (antipanNa ${ }_{v} \mathrm{Ch}, \mathrm{NaCh}$ ), which is known to label node structures in axons (Voas et al., 2007). After immunohistochemistry with a $\mathrm{NaCh}$ antibody, we observed that $\mathrm{NaCh}^{+}$node structures were regularly located in the PLL axons of the control larvae (Fig. 4A, arrows). However, $\mathrm{NaCh}^{+}$node structures were rarely located in the PLL axons of the MTZ-treated Tg(claudinK:gal4-vp16; uas:megfp; uas:nfsB-mcherry) larvae, indicating that the loss of myelin sheath disrupted node structures in the PLL axons (Fig. 4B, arrows). However, sulfasalazine-treated Tg(claudinK:gal4vp16;uas:megfp;uas:nfsB-mcherry) larvae showed an increased number of $\mathrm{NaCh}^{+}$node structures compared with the control larvae that recovered without sulfasalazine treatment, indicating that sulfasalazine treatment promoted remyelination with functional node structure formation (Figs. 4C-4E, arrows). 


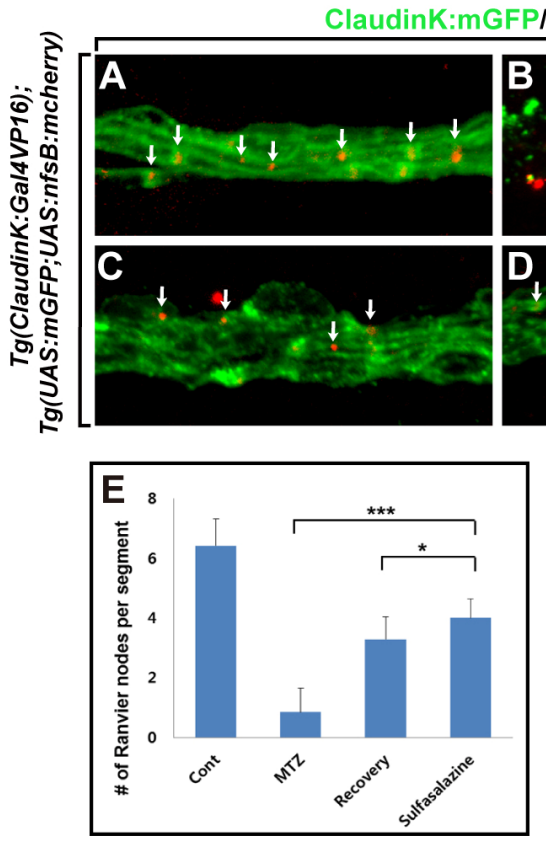

Fig. 4. Sulfasalazine-induced remyelination reconstructed myelin structure with node formation. All images are lateral views of PLL of $\mathrm{Tg}$ (claudink:gal4-vp16;uas: nfsB-mcherry; uas:mgfp) larvae with anterior to the left and dorsal to the top. Transgenic larvae were labeled with an anti-sodium channel (anti-panNa $\mathrm{N}_{\mathrm{v}} \mathrm{Ch}, \mathrm{NaCh}$ ) antibody to detect node structures (red color) in axons. Green color indicates ClaudinK:mGFP ${ }^{+}$myelin sheath. Transgenic larvae were treated with DMSO (A) and MTZ (B) at $5 \mathrm{dpf}$ for two days and recovered for one day in EM (C) and sulfasalazine solution (D). Arrows indicate $\mathrm{NaCh}^{+}$node structures. (E) Quantification of the number of node structures in the PLL of Tg(claudink:gal4-vp16;uas:nfsB-mcherry;uas:mgfp).Data were obtained from each of 11 transgenic larvae. Error bars represent s.d. $\left({ }^{* *} p<0.001,{ }^{*} p<0.05\right)$. Scale bar, $25 \mu \mathrm{m}$.
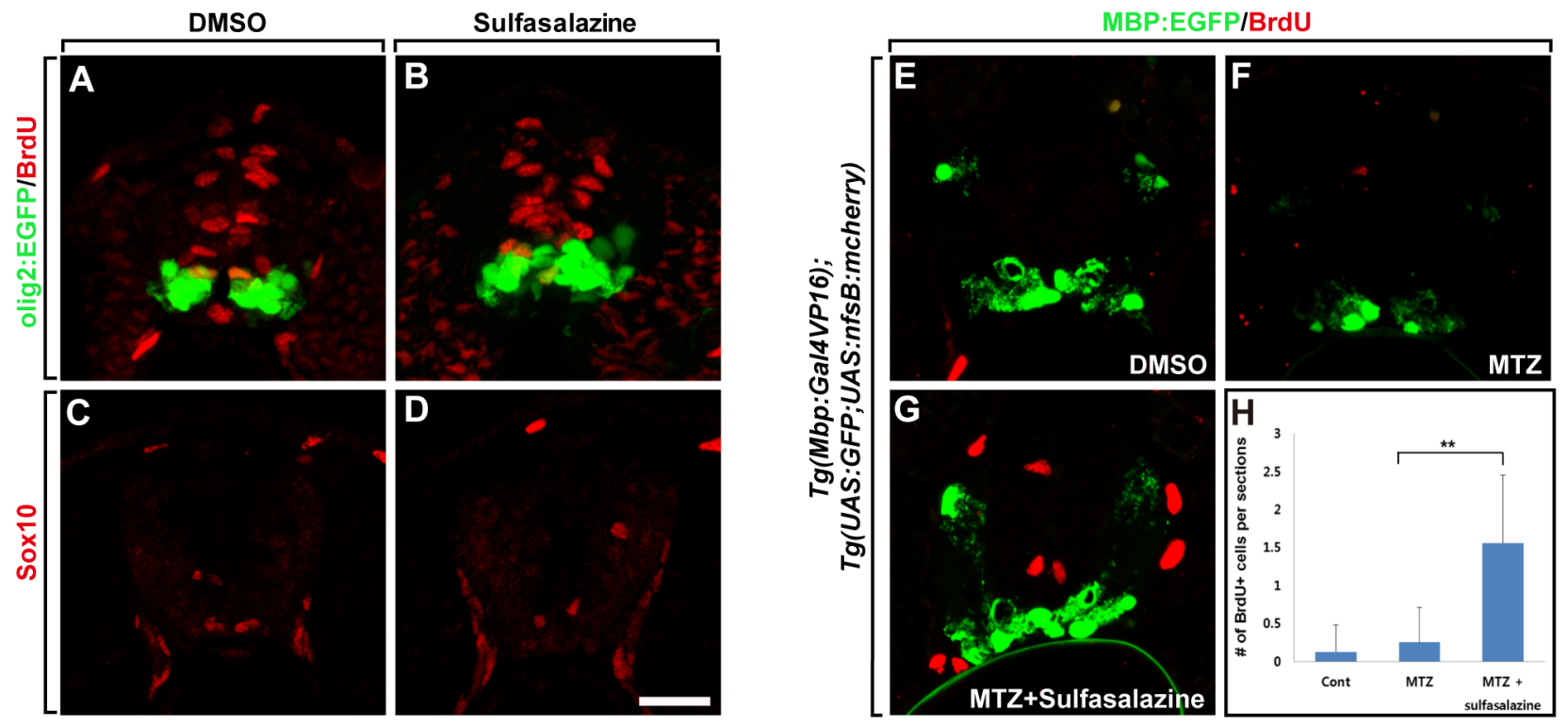

Fig. 5. Sulfasalazine promotes proliferation of neural precursors under demyelination status in the spinal cord of zebrafish larvae. (A-D) Transverse sections of the spinal cord of $T g$ (olig2:egfp) embryos treated with DMSO (A, C) and sulfasalazine (B, D) at 24 hpf. DMSO- (A) and sulfasalazine-treated (B) Tg(olig2:egfp) embryos were treated with BrdU for $20 \mathrm{~min}$ at $36 \mathrm{hpf}$ and labeled with an anti-BrdU antibody (red). DMSO(C) and sulfasalazine-treated (D) Tg(olig2:egfp) embryos were labeled with an anti-sox10 antibody (red) at 2 dpf. (E-G) Transverse sections of the spinal cord of $T g$ (mbp:gal4-vp16;uas:nfsB-mcherry;uas:gfp) larvae at $8 \mathrm{dpf}$ (dorsal at the top). Transgenic larvae treated with DMSO (E), MTZ (F), and MTZ in combination with sulfasalazine $(G)$ at $5 \mathrm{dpf}$ for three days were exposed to BrdU for $1 \mathrm{hr}$ at $8 \mathrm{dpf}$ and labeled with an antiBrdU antibody (red color). (H) Quantification of the number of BrdU ${ }^{+}$cells in the spinal cord of $T g$ (mbp:gal4-vp16;uas:nfsB-mcherry;uas:gfp) larvae. Data were obtained from each of the eight transgenic larvae. Error bars represent s.d. $\left.{ }^{* *} p<0.01\right)$. Scale bars: $20 \mu \mathrm{m}$.

\section{Sulfasalazine promotes precursor proliferation and} modulates the immune response

Our previous data showed that sulfasalazine treatment promoted regeneration of oligodendrocytes and Schwann cells and promoted subsequent remyelination in the NTR/MTZ- induced demyelination model. Therefore, we investigated the underlying mechanisms for the promotion of remyelination by sulfasalazine. To test whether sulfasalazine promoted proliferation of spinal cord precursors and thus the formation of oligodendrocyte precursor cells (OPCs) under normal conditions, we exposed wild-type $T g$ (olig2:egfp) embryos to DMSO and sulfa- 
salazine at $24 \mathrm{hpf}$, prior to OPC specification, and subsequently exposed the embryos to BrdU and performed immunohistochemistry with an anti-BrdU antibody at $36 \mathrm{hpf}$ to detect proliferation and with an anti-Sox10 antibody at $48 \mathrm{hpf}$ to detect OPCs. Since Tg(olig2: egfp) zebrafish express EGFP under the control of the olig2 promoter, EGFP ${ }^{+}$cells in this transgenic zebrafish mark neural precursors for motor neuron and oligodendrocyte formation (Shin et al., 2003). Anti-BrdU antibody labeling of the spinal cord sections showed that the number of $\mathrm{BrdU}^{+}$proliferating precursors is similar between the DMSOtreated control (Fig. 5A, cont:1.541 \pm 1.382 per sections) and sulfasalazine treated embryos (Fig. $5 \mathrm{~B}$, sulfasalazine:1.472 \pm 1.133 per sections), indicating that sulfasalazine treatment did not promote precursor proliferation in the wild-type CNS. Consistent with these data, the spinal cords of sulfasalazine-treated embryos showed a similar number of Sox $10^{+}$OPCs compared with that of DMSO-treated control embryos (Figs. 5C and 5D, cont: $2.812 \pm 1.108$, sulfasalazine:2.65 \pm 1.136 per sections). Next, we exposed $T g$ (mbp:gal4-vp16;uas:gfp;uas:nfsB-mcherry) larvae to DMSO, MTZ alone, and MTZ in combination with sulfasalazine at $5 \mathrm{dpf}$ for three days to induce demyelination, and after that, we treated demyelinated transgenic larvae with $\mathrm{BrdU}$ at $8 \mathrm{dpf}$ to test whether sulfasalazine promoted precursor proliferation during remyelination process. As shown in Fig. 5, sulfasalazine treatment in combination with MTZ increased the number of $\mathrm{BrdU}^{+}$proliferating precursor cells compared with that of the transgenic larvae treated with MTZ alone (Figs. 5F$5 \mathrm{H}$ ), indicating that sulfasalazine treatment promotes precursor proliferation under the demyelination status.

In the CNS of MS patients and in animal models of MS, substantial infiltration of macrophages and activation of microglia are observed and have been shown to be associated with disease severity (Lucchinetti et al., 2000; Prineas and Wright, 1978). In addition, inhibition of macrophage/microglia activation or a reduction in their density suppressed and attenuated progression of experimental autoimmune encephalomyelitis (EAE), a common immune-mediated animal model of MS (Bhasin et al., 2007; Heppner et al., 2005; Martiney et al., 1998). Altogether, these studies suggest that macrophages/microglia play a detrimental role in the remyelination process. Because sulfasa-
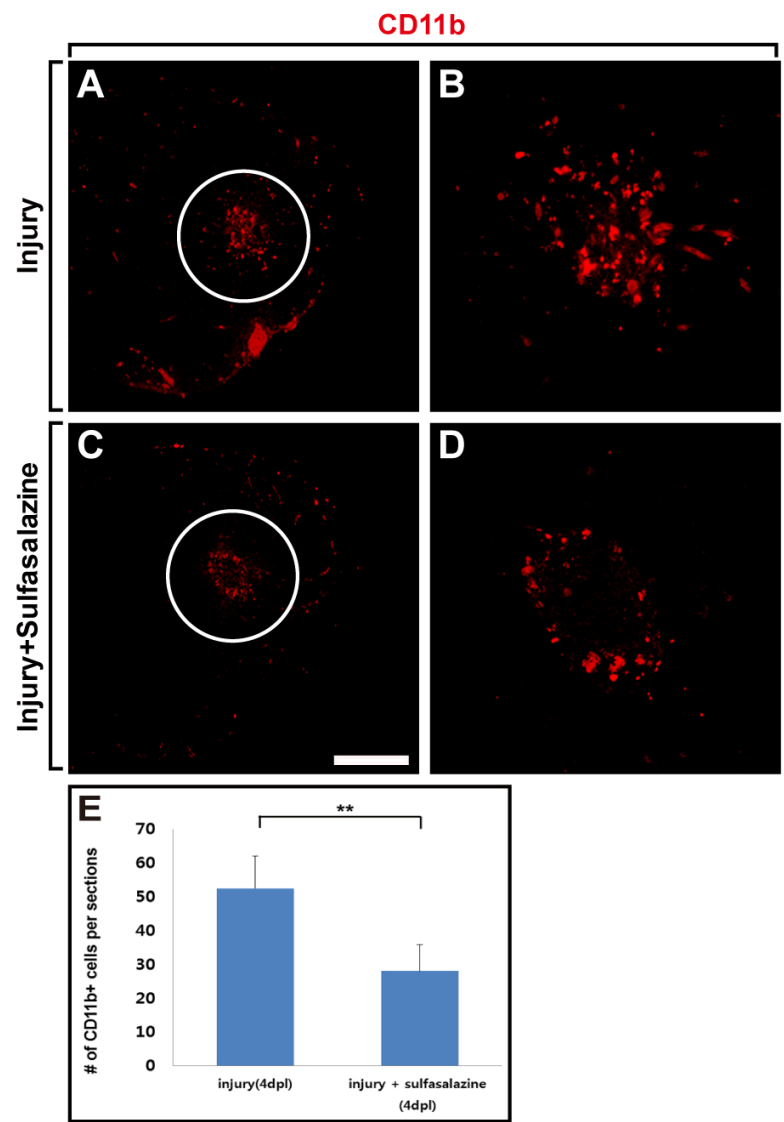
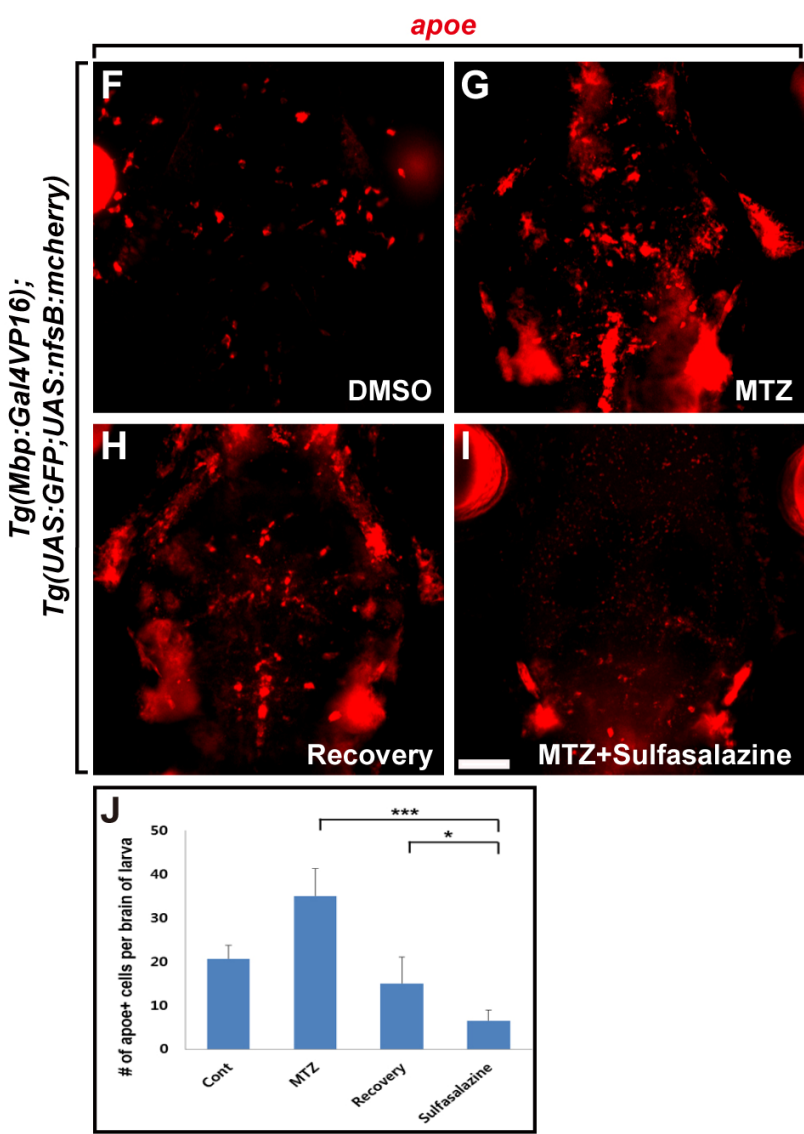

Fig. 6. Sulfasalazine modulates immune response by inhibiting recruitment of macrophages/microglia in the injured forebrain and demyelinated CNS. (A-D) Transverse sections of the injured telencephalic region of the three-month-old adult fish (dorsal at the top). Brain sections of the DMSO- $(A, B)$ and sulfasalazine-treated $(C, D)$ adult fish were labeled with anti-CD11b antibody to detect macrophages/microglia (red color) at $4 \mathrm{dpl}$ (days-post-lesion). (B, D) Higher magnification images of the circled areas in (A) and (C). (E) Quantification of the number of $\mathrm{CD}_{11 \mathrm{~b}^{+}}$macrophages/microglia at the stab lesion site. Data were obtained from each of five adult fish treated with DMSO and sulfasalazine, respectively. Error bars represent s.d. ( $\left.{ }^{* *} p<0.01\right)$. (F-J) Dorsal views of the brain of $T g$ (mbp:gal4-vp16;uas:nfsB-mcherry;uas:gfp) larvae with anterior at the top. Transgenic larvae were labeled by fluorescent in situ RNA hybridization with an apoe mRNA probe to detect macrophages/microglia (red color). Transgenic larvae were treated with DMSO (F) and MTZ (G) at $5 \mathrm{dpf}$ for two days and recovered for one day in EM $(\mathrm{H})$ and sulfasalazine solution (I). $(\mathrm{J})$ Quantification of the number of apoe ${ }^{+}$microglia in the brain. Data were obtained from each of the five larvae. Error bars represent s.d. $\left.{ }^{* \star *} p<0.001,{ }^{*} p<0.05\right)$. Scale bars: $200 \mu \mathrm{m}$ in $(A, B), 50 \mu \mathrm{m}$ in $(E-H)$. 
lazine is thought to be an anti-inflammatory and immunemodulating drug (Hoult, 1986; Peppercorn, 1984), we hypothesized that sulfasalazine treatment promotes remyelination process by modulating the behavior macrophages/microglia in the demyelination animal model.

To test this idea, we first induced injury in the forebrain of adult zebrafish and treated the injured brain with either DMSO or sulfasalazine. Next, we compared the number of macrophages/microglia in the injured forebrain of DMSO- and sulfasalazine-treated adult zebrafish following labeling with an antiCD11b antibody, which marks macrophages/microglia in zebrafish (Fantin et al., 2010). In the injured forebrain of sulfasalazine-treated adult zebrafish, the number of anti-CD11b-positive macrophages/microglia clearly decreased around the lesion site (Figs. 6C-6E) compared with that in the DMSO-treated control zebrafish forebrain (Figs. $6 \mathrm{~A}$ and $6 \mathrm{~B}$ ), indicating that sulfasalazine treatment reduced the number of macrophages/microglia in the injured forebrain.

To test whether sulfasalazine modulates macrophages/ microglia in the demyelination animal model, we next labeled Tg(mbp:gal4-vp16;uas:gfp;uas:nfsB-mcherry) larvae by fluorescent in situ RNA hybridization with an apoe mRNA probe, which was used to label macrophages/microglia (Tedde et al., 2008). After demyelination by MTZ treatment at $5 \mathrm{dpf}$ for three days, the number of macrophages/microglia markedly increased in the brain of MTZ-treated $T g$ (mbp:gal4-vp16;uas: gfp;uas:nfsB-mcherry) larvae compared with that in the DMSOtreated control, indicating that ablation of oligodendrocytes and subsequent demyelination recruited macrophages/microglia to the CNS (Figs. 6F and 6G). Interestingly, after sulfasalazine treatment, the number of macrophages/microglia are dramatically reduced in the brain of the MTZ-induced demyelination model compared with that of transgenic larvae that is recovered without sulfasalazine treatment (Figs. 6G-6I), indicating that sulfasalazine treatment reduced macrophages/microglia in the demyelinated brain. Altogether, these data suggest that promotion of oligodendrocyte regeneration and subsequent remyelination by sulfasalazine might be mediated by the immunomodulatory functions of sulfasalazine.

\section{DISCUSSION}

Macrophages are myeloid cells derived from hematopoietic stem cells in the bone marrow and circulate the peripheral vasculature to survey tissue environments for damage or infection (Miron and Franklin, 2014). Microglia are the resident macrophages in the CNS and are generated from erythromyeloid precursors in the embryonic yolk sac (Rawji and Yong, 2013). In this study, we have shown that sulfasalazine treatment promotes oligodendrocyte and Schwann cell regeneration and subsequent remyelination in the nervous system of a transgenic zebrafish model of demyelination, and sulfasalazine function in remyelination is mediated by modulation of the immune response. Thus, sulfasalazine treatment reduces the number of macrophages/microglia in the CNS of the transgenic zebrafish model of demyelination, suggesting that modulation of the immune response, especially macrophages/microglia, is important for the remyelination process.

In the CNS of MS patients, infiltration of macrophages and activation of resident microglia are observed, and these immune cells have been shown to be associated with disease severity (Lucchinetti et al., 2000; Prineas and Connell, 1979). From studies using several models of CNS demyelination such as EAE, cupizone- and lysolecithin-induced demyelination, macrophages/microglia have been shown to play a negative role in remyelination (Franklin and Ffrench-Constant, 2008; Miron and Franklin, 2014; Rawji and Yong, 2013). Inhibition of macrophages/microglia activation or a reduction in their density suppressed and attenuated EAE progression (Bhasin et al., 2007; Heppner et al., 2005; Martiney et al., 1998). Administration of the microglia inhibitor minocycline to the cuprizoneinduced demyelination model caused a decrease in demyelination and an improvement in motor coordination behavior (Pasquini et al., 2007; Skripuletz et al., 2010). In addition, treatment with corticosteroid enhanced remyelination associated with a reduction in the number of macrophages/microglia at the lesion site (Pavelko et al., 1998), and activation of macrophages/microglia using zymosan resulted in a loss of OPCs and oligodendrocytes in the lysolecithin-induced demyelination model (Schonberg et al., 2007). Altogether, these data indicate that macrophages/microglia play a negative role for remyelination in various models of demyelination in the CNS. Consistent with these studies, our data showed that reductions in macrophages/microglia are observed in the injured and demyelinated brain following sulfasalazine treatment, which resulted in the acceleration of the remyelination process (Fig. 6). This suggests that removal of macrophages/microglia from the demyelinated CNS promotes remyelination.

However, in contrast to its negative role in remyelination, several studies have shown that macrophages/microglia play a positive role in remyelination. First, in response to injury, OPCs near the lesion are activated from a normal quiescent state to a regenerative phenotype, which is the first step in remyelination, and macrophages/microglia are considered to be the major source of the factors that induce OPC activation (Franklin and Ffrench-Constant, 2008; Schonrock et al., 1998; Wilson et al., 2006). Second, after the activation and recruitment of OPCs to the demyelinated region, the next step in remyelination is the differentiation of OPCs into mature, myelinating oligodendrocytes. For the differentiation of OPCs, it is crucial to remove myelin debris that is generated during demyelination because CNS myelin contains proteins that inhibit OPC differentiation (Franklin and Ffrench-Constant, 2008; Kotter et al., 2006). In the cuprizone-induced demyelination model, removal of myelin debris by the phagocytic function of macrophages/microglia has been shown to be important for remyelination (Jurevics et al., 2002; Olah et al., 2012; Skripuletz et al., 2013). In the lysolecithin-induced demyelination model, depletion of macrophages reduced oligodendrocyte remyelination and delayed recruitment of OPCs to the lesion site by the lack of phagocytic function of macrophages, which is required for the removal of myelin debris (Kotter et al., 2001; 2005; 2006).

Because these reports and the discrepancies regarding whether the removal of macrophages/microglia promoted remyelination in our study, we reasoned that after demyelination, macrophages/microglia play a positive role in activating OPCs and removing myelin debris, but a prolonged presence of macrophages/microglia in a demyelinated region play a negative role in remyelination. In our experiment, we showed that the number of macrophages/microglia increased after demyelination by MTZ treatment. Thus, these cells have a chance to remove myelin debris and stimulate OPC activation for remyelination (Fig. 6F). Next, because we treated with sulfasalazine after demyelination occurred, sulfasalazine reduced the number of macrophages/microglia from the lesion after promoting recovery, and thus, the demyelinated CNS escaped from the negative effects caused by the sustained presence of macrophages/microglia. Our hypotheses are supported by the previous reports showing that the depletion of macrophages/microglia at the initial stage of demyelination significantly 
reduced remyelination but macrophage depletion at later stages in the remyelination phase does not change remyelination, suggesting that the beneficial role of macrophages/microglia was time-dependent (Kotter et al., 2001; 2005). Altogether, our data suggest that temporal regulation of the immune response of macrophages/microglia by sulfasalazine is important for the promotion of remyelination.

\section{ACKNOWLEDGMENTS}

We thank T. Becker for generous gift of an Tg(claudinK: gal4;uas:mGFP) zebrafish. This work was supported by a grant from the Samsung Advanced Institute of Technology (SAIT), Samsung Electronics Co.

\section{REFERENCES}

Asakawa, K., and Kawakami, K. (2008). Targeted gene expression by the Gal4-UAS system in zebrafish. Dev. Growth. Differ. 50, 391-399.

Bhasin, M., Wu, M., and Tsirka, S.E. (2007). Modulation of microglial/macrophage activation by macrophage inhibitory factor (TKP) or tuftsin (TKPR) attenuates the disease course of experimental autoimmune encephalomyelitis. BMC Immunol. 8, 10

Brend, T., and Holley, S.A. (2009). Zebrafish whole mount highresolution double fluorescent in situ hybridization. J. Vis. Exp. 25, pii: 1229.

Chung, A.Y., Kim, P.S., Kim, S., Kim, E., Kim, D., Jeong, I., Kim, H.K., Ryu, J.H., Kim, C.H., Choi, J., et al. (2013). Generation of demyelination models by targeted ablation of oligodendrocytes in the zebrafish CNS. Mol. Cells 36, 82-87.

Dale, J., Alcorn, N., Capell, H., and Madhok, R. (2007). Combination therapy for rheumatoid arthritis: methotrexate and sulfasalazine together or with other DMARDs. Nat. Clin. Pract. Rheumatol. 3, 450-458; quiz, following 478 .

Emery, B. (2010). Regulation of oligodendrocyte differentiation and myelination. Science 330, 779-782.

Fantin, A., Vieira, J.M., Gestri, G., Denti, L., Schwarz, Q., Prykhozhii, S., Peri, F., Wilson, S.W., and Ruhrberg, C. (2010). Tissue macrophages act as cellular chaperones for vascular anastomosis downstream of VEGF-mediated endothelial tip cell induction. Blood 116, 829-840.

Franklin, R.J. (2002). Why does remyelination fail in multiple sclerosis? Nat. Rev. Neurosci. 3, 705-714.

Franklin, R.J., and Ffrench-Constant, C. (2008). Remyelination in the CNS: from biology to therapy. Nat. Rev. Neurosci. 9, 839-855.

Heppner, F.L., Greter, M., Marino, D., Falsig, J., Raivich, G., Hovelmeyer, N., Waisman, A., Rulicke, T., Prinz, M., Priller, J., et al. (2005). Experimental autoimmune encephalomyelitis repressed by microglial paralysis. Nat. Med. 11, 146-152.

Hoult, J.R. (1986). Pharmacological and biochemical actions of sulphasalazine. Drugs 32 Suppl 1, 18-26.

Imai, M., Watanabe, M., Suyama, K., Osada, T., Sakai, D., Kawada, H., Matsumae, M., and Mochida, J. (2008). Delayed accumulation of activated macrophages and inhibition of remyelination after spinal cord injury in an adult rodent model. J. Neurosurg. Spine 8, 58-66.

Jurevics, H., Largent, C., Hostettler, J., Sammond, D.W., Matsushima, G.K., Kleindienst, A., Toews, A.D., and Morell, P. (2002). Alterations in metabolism and gene expression in brain regions during cuprizone-induced demyelination and remyelination. J. Neurochem. 82, 126-136.

Kim, H.T., Kim, I.H., Lee, K.J., Lee, J.R., Park, S.K., Chun, Y.H., Kim, H., and Rhyu, I.J. (2002). Specific plasticity of parallel fiber/Purkinje cell spine synapses by motor skill learning. Neuroreport 13, 1607-1610.

Kimmel, C.B., Ballard, W.W., Kimmel, S.R., Ullmann, B., and Schilling, T.F. (1995). Stages of embryonic development of the zebrafish. Dev. Dyn. 203, 253-310.

Kotter, M.R., Setzu, A., Sim, F.J., Van Rooijen, N., and Franklin, R.J. (2001). Macrophage depletion impairs oligodendrocyte remyelination following lysolecithin-induced demyelination. Glia 35, 204-212.

Kotter, M.R., Zhao, C., van Rooijen, N., and Franklin, R.J. (2005).
Macrophage-depletion induced impairment of experimental CNS remyelination is associated with a reduced oligodendrocyte progenitor cell response and altered growth factor expression. Neurobiol. Dis. 18, 166-175.

Kotter, M.R., Li, W.W., Zhao, C., and Franklin, R.J. (2006). Myelin impairs CNS remyelination by inhibiting oligodendrocyte precursor cell differentiation. J. Neurosci. 26, 328-332.

Lau, L.W., Keough, M.B., Haylock-Jacobs, S., Cua, R., Doring, A. Sloka, S., Stirling, D.P., Rivest, S., and Yong, V.W. (2012) Chondroitin sulfate proteoglycans in demyelinated lesions impair remyelination. Ann. Neurol. 72, 419-432.

Lee, Y., Morrison, B.M., Li, Y., Lengacher, S., Farah, M.H., Hoffman, P.N., Liu, Y., Tsingalia, A., Jin, L., Zhang, P.W., et al. (2012). Oligodendroglia metabolically support axons and contribute to neurodegeneration. Nature 487, 443-448.

Lucchinetti, C., Bruck, W., Parisi, J., Scheithauer, B., Rodriguez, M. and Lassmann, H. (2000). Heterogeneity of multiple sclerosis lesions: implications for the pathogenesis of demyelination. Ann. Neurol. 47, 707-717.

Martiney, J.A., Rajan, A.J., Charles, P.C., Cerami, A., Ulrich, P.C. Macphail, S., Tracey, K.J., and Brosnan, C.F. (1998). Prevention and treatment of experimental autoimmune encephalomyelitis by CNI-1493, a macrophage-deactivating agent. J. Immunol. 160, 5588-5595.

Marz, M., Schmidt, R., Rastegar, S., and Strahle, U. (2011). Regenerative response following stab injury in the adult zebrafish telencephalon. Dev. Dyn. 240, 2221-2231.

Miron, V.E., and Franklin, R.J. (2014). Macrophages and CNS remyelination. J. Neurochem. 130, 165-171.

Moharregh-Khiabani, D., Blank, A., Skripuletz, T., Miller, E., Kotsiari, A., Gudi, V., and Stangel, M. (2010). Effects of fumaric acids on cuprizone induced central nervous system de- and remyelination in the mouse. PLoS One 5, e11769.

Munzel, E.J., Schaefer, K., Obirei, B., Kremmer, E., Burton, E.A., Kuscha, V., Becker, C.G., Brosamle, C., Williams, A., and Becker, T. (2012). Claudin $\mathrm{k}$ is specifically expressed in cells that form myelin during development of the nervous system and regeneration of the optic nerve in adult zebrafish. Glia 60, 253270.

Olah, M., Amor, S., Brouwer, N., Vinet, J., Eggen, B., Biber, K., and Boddeke, H.W. (2012). Identification of a microglia phenotype supportive of remyelination. Glia $60,306-321$.

Pasquini, L.A., Calatayud, C.A., Bertone Una, A.L., Millet, V., Pasquini, J.M., and Soto, E.F. (2007). The neurotoxic effect of cuprizone on oligodendrocytes depends on the presence of proinflammatory cytokines secreted by microglia. Neurochem. Res. 32, 279-292.

Pavelko, K.D., van Engelen, B.G., and Rodriguez, M. (1998) Acceleration in the rate of CNS remyelination in lysolecithininduced demyelination. J. Neurosci. 18, 2498-2505.

Peppercorn, M.A. (1984). Sulfasalazine. Pharmacology, clinical use, toxicity, and related new drug development. Ann. Intern. Med. $101,377-386$

Plosker, G.L., and Croom, K.F. (2005). Sulfasalazine: a review of its use in the management of rheumatoid arthritis. Drugs 65, 18251849.

Prineas, J.W., and Wright, R.G. (1978). Macrophages, lymphocytes, and plasma cells in the perivascular compartment in chronic multiple sclerosis. Lab. Invest. 38, 409-421.

Prineas, J.W., and Connell, F. (1979). Remyelination in multiple sclerosis. Ann. Neurol. 5, 22-31.

Ransohoff, R.M. (2012). Animal models of multiple sclerosis: the good, the bad and the bottom line. Nat. Neurosci. 15, 1074-1077.

Rawji, K.S., and Yong, V.W. (2013). The benefits and detriments of macrophages/microglia in models of multiple sclerosis. Clin. Dev. Immunol. 2013, 948976.

Schonberg, D.L., Popovich, P.G., and McTigue, D.M. (2007) Oligodendrocyte generation is differentially influenced by toll-like receptor (TLR) 2 and TLR4-mediated intraspinal macrophage activation. J. Neuropathol. Exp. Neurol. 66, 1124-1135.

Schonrock, L.M., Kuhlmann, T., Adler, S., Bitsch, A., and Bruck, W. (1998). Identification of glial cell proliferation in early multiple sclerosis lesions. Neuropathol. Appl. Neurobiol. 24, 320-330.

Shin, J., Park, H.C., Topczewska, J.M., Mawdsley, D.J., and Appel, B. (2003). Neural cell fate analysis in zebrafish using olig2 BAC transgenics. Methods Cell. Sci. 25, 7-14. 
Silvestroff, L., Bartucci, S., Pasquini, J., and Franco, P. (2012). Cuprizone-induced demyelination in the rat cerebral cortex and thyroid hormone effects on cortical remyelination. Exp. Neurol. 235, 357-367.

Skripuletz, T., Miller, E., Moharregh-Khiabani, D., Blank, A., Pul, R., Gudi, V., Trebst, C., and Stangel, M. (2010). Beneficial effects of minocycline on cuprizone induced cortical demyelination. Neurochem. Res. 35, 1422-1433.

Skripuletz, T., Hackstette, D., Bauer, K., Gudi, V., Pul, R., Voss, E., Berger, K., Kipp, M., Baumgartner, W., and Stangel, M. (2013). Astrocytes regulate myelin clearance through recruitment of microglia during cuprizone-induced demyelination. Brain 136, 147-167.

Tedde, A., Cellini, E., Bagnoli, S., Sorbi, S., and Peri, A. (2008).
Mutational screening analysis of DHCR24/seladin-1 gene in Italian familial Alzheimer's disease. Am J. Med. Genet. B Neuropsychiatr. Genet. 147B, 117-119.

Voas, M.G., Lyons, D.A., Naylor, S.G., Arana, N., Rasband, M.N. and Talbot, W.S. (2007). alphall-spectrin is essential for assembly of the nodes of Ranvier in myelinated axons. Curr. Biol. $17,562-568$

Wilson, H.C., Scolding, N.J., and Raine, C.S. (2006). Co-expression of PDGF alpha receptor and NG2 by oligodendrocyte precursors in human CNS and multiple sclerosis lesions. J. Neuroimmunol. $176,162-173$

Zenlea, T., and Peppercorn, M.A. (2014). Immunosuppressive therapies for inflammatory bowel disease. World J. Gastroenterol. 20, 3146-3152. 